\title{
LE MAGAZINE MONTREALAIS VICE VERSA : LES OPINIONS DES LECTEURS
}

Gerardo ACERENZA

Università degli Studi di Trento (Italie)

\begin{abstract}
En): No other cultural magazine than Vice Versa has attracted attention of critics in Quebec, Canada and elsewhere in the world. Since its appearance on the Montreal's cultural scene, there is no count of the total number of scientific articles, communications at conferences and master's theses dedicated to this magazine. Each scientific work on "migrant literature" refers to the journal Vice Versa. We have the impression that everything, or almost everything, has already been said on this magazine. The aim of this article is to understand the feelings of the readers of Vice Versa, the feelings of the magazine's subscribers, but also the feelings of occasional readers who sent letters for publication in the Letters to the Editor's section. This is, in our view, the only aspect that has not been dealt with so far.
\end{abstract}

Keywords (En): Montreal; magazine; Vice Versa; identity; letter; reader opinion.

Mots-clés (Fr) : Montréal ; magazine ; Vice Versa ; identité ; lettre ; opinion du lecteur.

\section{Introduction}

Dès la parution du premier numéro, le magazine culturel Vice Versa a attiré l'attention de la critique au Québec, au Canada et également ailleurs dans le monde et on ne compte plus aujourd'hui le nombre d'articles scientifiques, de communications présentées à des colloques et de mémoires de maîtrise qui portent sur cette revue. Chaque travail scientifique sur la «littérature migrante» fait référence à Vice Versa, car, pour certains, c'est dans ses pages que le poète d'origine haïtienne Robert BERROUËT-ORIOL (1986 : 20-21) utilise pour la première fois la formule « littérature migrante ». On a même consacré à ce magazine deux colloques, l'un en Italie en 2005, dont les actes sont parus en 2006 sous la direction de Anna Paola MossetTo avec le titre Le Projet transculturel de Vice Versa, et l'autre au Québec, en 2007, colloque organisé à l'Université Concordia pour commémorer les dix années de la disparition de la revue. Les actes ont paru en 2010 sous le titre $L a$ Transculture et Vice Versa, sous la direction de Fulvio Caccia. Les deux ouvrages soulignent dans les titres la vocation «transculturelle» de ce périodique. En effet, dans un article fort intéressant sur les revues culturelles québécoises, Michel NAREAU (2011: 14) a bien souligné que la revue Vice Versa est la seule revue «transculturelle» au Québec et également l'une des revues les plus commentées avec les revues Liberté, Parti pris et Cité libre.

Nous avons ainsi l'impression que tout ou presque tout a déjà été dit sur ce magazine montréalais. Il s'agit toutefois d'une reconnaissance tardive puisqu'une bonne partie de l'intelligentsia québécoise a adressé, lors de la parution des premiers numéros, des critiques très sévères à l'égard des auteurs du périodique et de leurs textes, qui prônaient pour le Québec une vision très souvent fédéraliste et non pas souverainiste. Un grand nombre d'intellectuels québécois n'avaient pas encore fait 
le deuil de la défaite référendaire de 1980 et la société entière était toujours caractérisée par des tensions inter-ethniques. De plus, l'utilisation de trois et parfois de quatre langues différentes menaçait, aux yeux de certains, l'unilinguisme et la suprématie du français dans la Belle Province. Pierre Nepveu, par exemple, qui a également collaboré au magazine en y publiant de nombreux textes, soulignait en 2005, lors du colloque de Rome, qu' " on ne peut négliger le fait qu'en voulant jouer de tous les registres, de tous les genres, de tous les contenus en plusieurs langues, Vice Versa a présenté une personnalité essentiellement composite, tous azimuts, qui ne pouvait que dérouter une bonne partie du public intellectuel». Selon lui, les intellectuels québécois sont « demeurés à distance de la revue, y voyant surtout un curieux phénomène, un étrange objet dont on ne savait trop que faire ». Bref, concluait Pierre NEPVEU, le magazine était « trop beau, trop léché aux yeux de certains, trop éclaté, trop dans le genre mosä̈que ou collage, ce qui était un peu louche et pouvait laisser croire à une certaine frivolité, à un effet plus médiatique que véritablement intellectuel et profond » (NEPVEU, $2006: 83-84$ ).

Régine Robin, quant à elle, soulignera que la raison du manque d'intérêt des Québécois pour ce magazine tient essentiellement au «démon du nationalisme ethnique» :

\footnotetext{
Dans cette quête [pour fonder un véritable lieu culturel], notre échec fut très productif. Même si nous n'avons pas été compris (le démon du nationalisme qu'on l'appelle ethnique ou civique a vite repris l'intelligentsia québécoise), nous avions développé une pensée qui a l'avenir pour elle dans tous les pays qui sont devenus peu ou prou des pays d'immigration. (MosseTTO, $2006: 16$ ).
}

Mais au-delà des critiques officielles formulées par l'intelligentsia québécoise, «déstabilisée » par l'arrivée de cette nouvelle revue dans le panorama culturel montréalais, nous avons voulu comprendre quel était le sentiment des lecteurs de Vice Versa, des lecteurs abonnés à la revue, mais également des lecteurs occasionnels qui adressaient des lettres à la rédaction pour qu'elles soient publiées dans la rubrique du «Courrier des lecteurs ». Il s'agit, d'après nous, du seul aspect qui n'ait pas encore été traité jusqu'à nos jours. Tout d'abord, en guise d'introduction à cet article, nous proposerons une brève histoire de ce magazine et de ses acteurs principaux. Ensuite, nous commenterons le courrier des lecteurs des 53 numéros parus de 1983 à 1996 et nous allons tenter de répondre à la question suivante : quelles sont les réactions des lecteurs québécois ou des lecteurs tout court lors de l'apparition de ce périodique plurilingue, unique en son genre, dans le panorama littéraire du Québec de l'époque ? Cette question est centrale, vu que le premier numéro, avec un éditorial et des articles en trois langues (italien, français et anglais), est lancé au cours de l'été 1983, c'est-à-dire dans un contexte postréférendaire où le débat autour de la souveraineté du Québec et de la centralité de la langue française n'était pas encore achevé, mais battait son plein.

\section{Le Courrier des lecteurs}

Nous avons choisi de nous consacrer au courrier des lecteurs, car il s'agit d'un espace très particulier au sein d'un magazine culturel et qui diffère de celui d'un quotidien ou d'un forum en ligne. Il s'agit en effet d'un espace hybride dans lequel 
la parole des lecteurs, qui proviennent d'horizons divers, peut apporter une contribution aux débats lancés par les auteurs des articles. D'habitude, les lettres reçues par un périodique expriment l'opinion personnelle du lecteur qui peut transmettre le contentement ou le mécontentement sur le choix des sujets traités par le comité éditorial, ou sur les opinions exprimées par les auteurs des articles. L'auteur peut être aussi bien un lecteur occasionnel qu'un lecteur habituel, c'est-àdire un abonné du magazine. Ces lettres peuvent parfois poser tout simplement des questions aux auteurs des articles et attendre une réponse, ce qui fait qu'elles pourraient en outre nourrir et relancer certaines réflexions qui apparaissent plus intéressantes que d'autres. Très souvent, les opinions des lecteurs sont prises en comptes par la rédaction pour mieux répondre aux attentes des abonnés. Les interventions des lecteurs peuvent alors, de manière générale, donner des indications sur la vitalité de la revue et sur l'intérêt qu'elle suscite au sein de son public. Dans le cas de Vice Versa, l'on suppose qu'il s'agit d'un lecteur suffisamment cultivé, puisqu'il achète et lit un périodique culturel et plurilingue ne proposant pas de sujets tirés de la vie de tous les jours, à l'instar d'une rubrique des chiens écrasés. Toutefois, d'un point de vue formel, le lecteur doit en même temps posséder un esprit de synthèse, car les lettres trop longues risquent de ne pas être publiées.

Nous avons analysé tout le courrier des lecteurs publié par Vice Versa à partir du premier numéro, paru en 1983, jusqu'au dernier numéro de la première vie du périodique paru en automne 1996, pour un total de 53 numéros. Comment les lecteurs ont-ils salué l'arrivée de ce magazine unique en son genre sur la scène culturelle québécoise? Puisque le périodique est publié à Montréal, les commentateurs sont-ils tous des Québécois, ou bien habitent-ils également d'autres provinces canadiennes ? Sont-ils en majorité des Néo-Québécois qui appartiennent aux communautés allophones? Quelle est la nature des commentaires de leurs interventions sur la scène publique ? Il s'agit en effet, répétons-le, d'une parole privée qui fait irruption dans le débat public pour être écoutée, pour faire entendre son point de vue.

\section{Avez-vous dit Vice Versa?}

Avant de voir de près la nature de ces lettres, un détour sur l'histoire de ce magazine nous permettra de mieux le situer dans le contexte québécois de l'époque, c'est-à-dire la période des années quatre-vingt et quatre-vingt-dix. Le premier numéro du magazine, présenté à la Bibliothèque Nationale de Montréal, apparaît dans le panorama littéraire québécois comme un objet volant non identifié au cours de l'été 1983. Le format n'est pas vraiment pratique, il est « inclassable », puisque les pages mesurent $28 \mathrm{~cm}$ sur $42 \mathrm{~cm}$, beaucoup trop grandes pour un magazine culturel. Toutefois, elles permettent d'insérer de grandes photos et des publicités qui ne passent pas inaperçues. Gianni Caccia, à l'époque directeur artistique, s'inspire pour le graphisme du magazine des revues pop art américaines. Le prix de vente est fixé à $1 \$$, la qualité des pages ressemble à celle des quotidiens papier et le titre latin Vice Versa permet d'être lu très facilement dans les trois langues utilisées par les auteurs pour la rédaction des articles : le français, l'anglais et l'italien. Avec le titre principal, apparaît sur la première page le syntagme nominal «magazine 
transculturel » et l'adjectif « transculturel » déclenchera tout une série de réactions, de questionnements et de commentaires critiques. Qu'entendait-on à l'époque par cet adjectif? De quelle manière qualifiait-on le magazine vu que les termes interculturel et multiculturel existaient déjà ?

La «Lettre de l'éditeur» du premier numéro paru en été 1983 souligne sur plusieurs passages la nouveauté que le magazine aimerait représenter dans le panorama québécois des périodiques culturels. En effet, les premiers mots des textes en trois langues sont «Vice Versa è una nuova rivista; Renouvellement ; A new magazine ?». Toutefois, ni le mot «transculture », ni l'adjectif «transculturel», n'apparaissent dans les trois versions de l'éditorial. En revanche, les rédacteurs utilisent le syntagme nominal « espace interculturel » lorsqu'ils invitent les lecteurs à faire parvenir des suggestions ou des critiques :

Renouvellement. Tel est le maître mot de cette première édition de Vice Versa, un magazine qui a déjà une demi-vie derrière lui. En effet, les lecteurs qui ont suivi notre activité éditoriale depuis le début des Quaderni culturali auront remarqué la transformation que reflète ce présent numéro. Ils la noteront bien sûr dans le nouveau format et la conception graphique également dans les sujets abordés, sa composition linguistique, dans son équipe éditoriale et dans ses collaborateurs. Avec Vice Versa, nous continuons donc notre intervention sur le terrain que représente le point de jonction de divers univers culturels. Nous voulons enquêter, nous voulons retracer, nous voulons critiquer, nous voulons rire, nous voulons imaginer; tout ceci à travers un modèle souple, qui peut porter tant la marque de l'intellectuel inspiré, de l'émigrant fraîchement débarqué ou du Québécois de vieille souche. Un modèle souple et mobile dont les frontières sont vastes comme celles de l'émigration. Bien sûr, cet effort part d'abord et avant tout des besoins des rédacteurs qui ont la plupart un pied dans la réalité italo-québécoise et les deux autres dans celle nord-américaine. [...] (VICE VERSA $\left.\mathrm{n}^{\circ} 1,1983: 3\right)$

Dans le long extrait de l'éditorial que nous venons de citer, trois points importants se détachent parmi tant d'autres : le premier souligne que la ville de Montréal et le Québec sont des lieux de rencontre et de contact de plusieurs cultures et par conséquent le terrain privilégié d'intervention du nouveau magazine. Le deuxième point concerne l'ouverture à des collaborateurs venant de tout horizon : « l'intellectuel inspiré », l'émigrant qui vient d'arriver au Québec et également le Québécois de vieille souche. Le troisième point considère le rôle des futurs lecteurs qui sont invités à suggérer des idées à la revue ou bien à la critiquer par le moyen de lettres envoyées à la rédaction. Le dernier passage de la longue citation mérite d'être cité intégralement: "Son rayonnement [de Vice Versa] réside surtout dans l'implication du lecteur. De lui dépend l'essentiel du succès de ce projet. Vos suggestions et vos critiques nous aideront justement à mieux identifier cet espace interculturel et à vérifier dans quelle mesure le besoin de la revue reflète un besoin collectif $»$. Et c'est sur ce troisième point que nous allons diriger notre attention dans la suite de cet article.

Les membres fondateurs de la revue, Lamberto Tassinari, Antonio D'Alfonso et Fulvio Caccia, avaient déjà travaillé ensemble depuis quelques années dans les bureaux de la rédaction des Quaderni culturali, une revue bimestrielle trilingue publiée par l'" Association de culture populaire ». Comme le souligne Lamberto TASSINARI (1989: 57), deux options se profilaient au sein du groupe fondateur: une première hypothèse considérait la possibilité de continuer la mission de la revue 
Quaderni culturali, qui était politiquement engagée à gauche et qui s'adressait surtout aux immigrés italiens de deuxième génération avec l'intention d'appuyer également leurs revendications syndicales, tout en proposant une profonde réflexion sur la condition sociale de l'immigré. Selon les partisans de cette première option, la revue aurait dû être distribuée gratuitement au sein des communautés immigrées, notamment au sein de la communauté italienne. L'autre option envisageait plutôt de s'ouvrir à la société québécoise dans sa globalité, de ne plus rester enfermés dans l'espace des communautés immigrées du Québec, mais d'orienter la réflexion sur une notion plus large, c'est-à-dire celle de la «transculture », que TASSINARI expliquera par la suite avec ces mots : «Le trans [...] proposé par Vice Versa signifiait traversée, passage métamorphose continue de l'identité : perte et gain sans arrêt, osmose » (TASSINARI, $2006: 23$ ). Une revue plus moderne donc, qui ne devait pas vivre seulement grâce aux subventions du gouvernement, mais qui aurait dû trouver un soutien financier également auprès des privés. Cette dernière option fut choisie par les membres fondateurs de Vice Versa.

\section{Les lettres envoyées à la rédaction}

Quelle est la nature des opinions des lecteurs sur le magazine exprimées par le moyen de lettres envoyées à la rédaction ? D'emblée, il faut préciser que nous avons trouvé très peu de courrier des lecteurs et cela est peut-être dû à la nature même du magazine, c'est-à-dire une publication culturelle bimensuelle qui ne traite pas de sujets d'actualité comme les quotidiens d'information qui paraissent tous les jours. De plus, dans les années quatre-vingt et jusqu'à l'année quatre-vingt-seize, le courriel n'existait pas ou n'était pas encore très répandu. Il fallait donc écrire une lettre à la main ou la taper à la machine, acheter l'enveloppe et le timbre et la mettre dans une boîte aux lettres, ce qui pouvait décourager un grand nombre de lecteurs du magazine qui auraient voulu réagir immédiatement à certains propos contenus dans les articles. Bref, dans les cinquante-trois numéros parus entre 1983 et 1996 que nous avons consultés, nous n'avons trouvé que vingt-quatre lettres de lecteurs adressées à la rédaction. Toutefois, cinq de ces vingt-quatre lettres ne sont pas de véritables lettres, mais plutôt des annonces de lecteurs à la recherche d'une âme sœur ou d'une location d'appartement, des annonces publiées dans la section « Le courrier du Vice » du numéro qui a pour titre «L'Italie, une manière d'être ». Ces lettres ne peuvent pas fournir d'informations analysables dans le cadre de notre étude. En voilà deux à titre d'exemple...

Jeune professionnel trilingue, cinéphile et ambitieux, 27 ans, ressemblant à Mickey Rourke dans 9 1/2 weeks (oui, oui c'est vrai!) cherche jeune Italienne entre 23-26 ans, spigliata avec l'intensité secrète de Monica Vitti, les yeux d'Ornella Muti, la passion d'Anna Magnani et la désinvolture de Stefania Sandrelli. Aventurières s'abstenir. Écrivez au C.P. n¹1. Réponse garantie.

Transculturel dans la trentaine cherche transculturelle fin de la vingtaine pour partager vision. C.P. $\mathrm{n}^{\circ}$ 24. (VICE VERSA, 1986, $\left.\mathrm{n}^{\circ} 16: 47\right)$.

Nous pouvons bien nous demander pourquoi c'est seulement dans ce numéro que les lecteurs du magazine trouvent de telles annonces! Nous sommes convaincu 
qu'il s'agit, sans aucun doute, d'une mauvaise stratégie de la rédaction pour avoir une source supplémentaire de financement, qui a peut-être été critiquée par les membres mêmes du comité éditorial.

Quoi qu'il en soit, des dix-neuf lettres retenues, treize sont en français, cinq sont en anglais et une seulement en italien. Les lettres sont toutes signées avec prénom et nom, sauf une qui est signée seulement par le prénom «Ivan » (VICE VERSA, 1985, $\mathrm{n}^{\mathrm{o}} 2: 3$ ). Seulement quelques-unes de ces lettres indiquent le lieu de résidence du signataire. La page de publication de ces lettres varie selon le numéro du magazine. Le plus souvent elles sont publiées dans les pages finales, surtout dans les derniers numéros, mais également dans les premières pages, surtout pour les premiers numéros.

Qui sont ces lecteurs ayant senti le besoin d'envoyer leurs commentaires à Vice Versa? À lire les noms des signataires, nous supposons qu'ils sont surtout des Québécois, qui résident au Québec, ou qui habitent à l'extérieur de la Belle Province, c'est-à-dire dans le Canada anglophone, mais il y a également des anglophones, des Italo-Canadiens et un Italien d'Italie qui serait tombé par hasard sur un numéro de la revue. Certains d'entre eux indiquent également leur profession. Voici leurs noms :

Patrick Coppens ; Jacques Dufresne; Pierre Boucher, Montréal ; Elettra Bedon; Ivan ; Gilbert Martin, chômeur et diplômé 5802, rue Waverly, app. 4, Montréal ; Catherine Clément ; Jenny Signoretti ; Albert Maronani ; Lucie Dallaire, Saint-Jean-sur-Richelieu ; Claire Simard, directrice, service des expositions ; Robert Montplaisir, Directeur de District-Division canadienne Metro-Goldwyn-Mayer United Artists ; Brigitte De Souza à Vanier, Ontario ; Heroldo Pereira ; Jacques Brisson; Pierre Boucher ; Anatol Orlovsky, Montréal ; Michele Pipan, Trieste, Italy ; Davide Di Labio, Ottawa.

Le premier commentaire que l'on peut hasarder à la suite de ces premières données est assez net: les lecteurs ne réagissent pas souvent aux sujets proposés dans les articles du magazine et ceux qui écrivent à la rédaction sont en majorité des francophones québécois, une minorité d'anglophones et très peu de lecteurs d'origine italienne. Cela peut apparaître étrange, vu que le berceau du magazine se situait au sein de la communauté italienne de Montréal.

Toutefois, ce qui nous intéresse le plus, c'est la nature des commentaires adressés au magazine et nous pouvons également souligner que nous n'avons pas trouvé beaucoup de commentaires intéressants. Nous nous attendions à trouver beaucoup de lettres des lecteurs dès le deuxième numéro publié, puisque Vice Versa représentait une réelle nouveauté dans le panorama des périodiques culturels. Cependant, le magazine n'a reçu et publié que deux lettres dans le deuxième numéro et il fallait attendre les numéros 10 et 11, parus deux ans plus tard, en 1985, pour trouver la troisième, la quatrième et la cinquième lettre des lecteurs. L'appel lancé par Lamberto Tassinari dans l'éditorial du premier numéro n'a pas vraiment été entendu.

Un lecteur québécois, qui s'appelle Jacques Dufresne, est ravi de constater l'effort de mettre la culture italienne à la portée des Québécois et semble satisfait puisqu'il avoue avoir testé entre autres choses ses compétences de compréhension écrite de la langue italienne... 
Vice Versa est un journal qui me paraît à la fois original et utile. Ce qui m'intéresse le plus c'est l'effort que vous semblez vouloir faire pour mettre la culture italienne à notre portée. Mon premier réflexe a été de lire la partie italienne et de vérifier ma traduction à l'aide du texte en regard. Quelle joie, je crois avoir bien compris le texte de Ramirez. Jacques Dufresne. (VICE VERSA, 1983, $\mathrm{n}^{\circ} 2: 2$ ).

Il faut préciser néanmoins que, mis à part les éditoriaux, les articles de la revue n'étaient pas des traductions, mais qu'ils étaient écrits et publiés en anglais, en français et en italien. Cette richesse linguistique était vue par certains Québécois comme une atteinte à la sacralité du français. Mais pour Vice Versa cela signifiait faire revivre une langue, l'italien, qui était pour certains immigrés la langue d'un passé perdu à jamais. La présence de l'italien détournait d'une certaine manière l'attention portée sur le rapport conflictuel français-anglais et présentait les trois langues sur un même niveau d'équivalence ce qui pouvait déplaire, peut-être, à certains puristes. Dans ce même numéro, la deuxième lettre reçue et publiée apparaît d'emblée cryptique...

Dans ce pays des mots traduits, paradoxalement si pauvre en signe, un mouvement d'humeur - à la limite - est une preuve qui trahit l'amitié. Surtout si je ne suis rien. Fin des grands airs. De l'air, de l'air. Et vice ne versera jamais dans la manie. Aujourd'hui avance sur la modernité et ses œsophages. Patrick Coppens. (VICE VERSA, 1983, nº $2: 2$ ).

Quel est le sentiment exprimé par l'auteur de cette brève lettre ? À lire dans la pensée de ce lecteur de la revue qui s'appelle Patrick Coppens, nous croyons déceler derrière ses mots et ses ellipses, une forme de reconnaissance à l'égard de ce magazine qui apporte un nouvel «air», de nouvelles idées en proposant une troisième langue comme l'italien. Le Québec est ressenti par ce lecteur comme une «société-œsophage » qui monopolise le débat culturel et linguistique et efface toutes les formes de diversités. Vice Versa est pour ce lecteur un signe de la « modernité », une alternative à la «manie » ambiante, c'est-à-dire aux débats politiques pour ou contre la souveraineté qui caractérisaient la société québécoise de l'époque.

Pour lire encore une lettre qui exprime le contentement du lecteur concernant la qualité du périodique, il faut attendre deux ans, car elle sera publiée dans le troisième numéro de l'année 1985 :

Au collectif de Vice Versa, pour que votre magazine poursuive son éclatante présence sur le tissu social, je m'empresse de renouveler mon abonnement. Qu'il me soit permis, par la présente, de vous adresser des félicitations à l'endroit de deux initiatives qui ont vu le jour en 84 : votre participation au colloque «L'Italie : le philosophe et le gendarme » ainsi que l'annonce de collaborateurs(trices) extérieurs (es) pour les prochains numéros. Une question continue cependant à hanter mon esprit : votre collectif a-t-il songé à laisser place à un « espace libre » pour commenter, discuter soit ses articles antérieurs soit des observations de nature « transculturelle»? Un lecteur assidu. Pierre Boucher, Montréal. (VICE VERSA, 1985, n $3: 2$ ).

Ce lecteur assidu, après avoir félicité les initiatives scientifiques du magazine, s'empresse de suggérer aux rédacteurs de Vice Versa de réserver plus d'espace dans le magazine aux lecteurs, de leur permettre de proposer plus de commentaires sur la 
nature des textes publiés. Ce commentaire se fait l'écho de l'éditorial de Lamberto Tassinari publié dans le premier numéro dans lequel ce dernier sollicitait la participation du public aux débats « transculturels ».

Nous aimerions commenter encore deux lettres envoyées à la rédaction du magazine. La première par une Torontoise d'origine italienne, publiée en 1990, qui précise d'emblée connaître les trois langues utilisées dans les pages Vice Versa:

I read with interest the article on your magazine, Vice Versa, in the Globe and Mail last July 29. Being of Italian parentage, and fluent in English, Italian and (rusty) French, I was intrigued by your vision of transculturalism. Your statements prompted me to buy the magazine, and I was not disappointed. (Vice Versa, $\mathrm{n}^{\circ} 26$ ) I found the literary submissions stimulating. The mix of languages and experiences work well together. Perhaps what struck me most was the realization that I was pan of a rich and diverse culture, though I'd never before seen it presented (packaged) as well as in your magazine. Up until this point, I had taken the insular and culturally segregated lifestyle of Toronto (where I was educated and where I've lived all my life) largely for granted, and had accepted it as the Canadian status quo. It is refreshing to learn that this is not the case. How may I obtain a subscription to your magazine? Do you accept unsolicited manuscripts of fiction/poetry? I look forward to your prompt response and I wish you continued success in your publication. Jenny Signoretti. (VICE VERSA, 1990, $\mathrm{n}^{\circ} 28: 58$ )

Néanmoins, ce qui est intéressant dans ce commentaire, c'est la découverte que la lectrice avoue avoir faite en lisant le numéro 26 du magazine. Vice Versa lui a permis de comprendre que le Canada n'était pas une société « organisée en univers culturels séparés » comme la ville de Toronto où la lectrice est née et où elle a toujours vécu, mais qu'elle faisait partie d'une «culture riche et diverse » qui était très bien "présentée dans la revue». À travers ce commentaire, le magazine apparaît comme le miroir d'une société multilingue et hétérogène qui réussit tout de même à établir des liens entre ses multiples réalités culturelles, à les faire dialoguer pour qu'elles se connaissent mieux. Cette lettre traduit à merveille, d'après nous, le projet transculturel de Vice Versa.

De la même manière, dans la dernière lettre que nous aimerions commenter, on sent que la lectrice Brigitte De Souza, qui habite Vanier en Ontario, exprime sa reconnaissance envers le magazine qui lui permet « d'avancer dans ses réflexions ». D'après elle, les membres du collectif Vice Versa, à la différence des «autres » rédacteurs de journaux ou de revues, "osent s'affirmer, prendre position, expliquer ». Elle fait surtout référence aux idées politiques véhiculées par le magazine qui ne cache pas son engagement contre la souveraineté du Québec...

Enfin, des gens qui osent s'affirmer, prendre position, expliquer (tandis que d'autres continuent à nous prendre pour des cons incapables de penser!) [...] Et vous annoncez vos couleurs en introduction (avis à ceux qui vivent sur les a priori). Tiens, tiens, un journal qui s'engage politiquement sans tomber dans une sauce délayée style B.B.Q. Ça existe encore? [...] Et pourtant, je ne partage pas toutes vos idées politiques. Par exemple, je suis pour l'indépendance du Québec (ou la souveraineté? On pourrait s'interroger sur le sens de ces deux mots et sur ce qu'ils recouvrent). Mais ce qui me plaît dans la revue, c'est que vous ne me servez pas un discours tout mâché en me disant: voilà, c'est comme ça qu'il faut penser (ce que font la majorité des quotidiens et des revues existants). Vous m'aidez à avancer dans mes réflexions, soit en me donnant des arguments qui viennent renforcer mes opinions, soit en m'ouvrant des horizons ou encore en me faisant accepter certaines choses que je refusais parce qu'alors, je "comprends" pourquoi. Merci pour cela. Je vais continuer à vous lire - plus que jamais - à vous soutenir - (je ne sais trop 
comment... en me réabonnant, en parlant de la revue, en la citant) - et vous me donnez le goût de prendre réellement part aux débats actuels. [...] Bien à vous. Bonne suite. J'ai hâte de lire le prochain numéro. Brigitte De Souza à Vanier. Ontario. (VICE VERSA, 1995, nº 49 : 58)

Aux yeux de cette lectrice, Vice Versa joue un rôle important, car elle « ouvre des horizons » et présente la réalité politique du Québec avec un « discours autre » par rapport aux discours existants. Ce que la lectrice souligne, c'est que Vice Versa tente en quelque sorte de redéfinir l'identité québécoise, de dépasser la vision d'un Québec fossilisé dans son idée nationaliste, de pureté et d'unilinguisme où il n'y a pas de place pour les autres. Avec sa mission transculturelle, Vice Versa se propose ainsi de questionner l'identité québécoise en la présentant comme une société « en devenir », comme un laboratoire. Le but du magazine était ainsi de participer et de faire participer ses lecteurs au devenir même de la société en y ajoutant un point de vue autre, un « discours qui n'était pas tout mâché » comme le souligne la lectrice.

\section{Conclusion}

Aujourd'hui, le magazine vit une deuxième vie et il est publié seulement en ligne. À partir de janvier 2014, la revue paraît tous les mois avec un nouveau numéro. Fulvio Caccia et Lamberto Tassinari continuent à proposer leurs réflexions sur l'actualité culturelle du Québec et d'ailleurs, mais nous avons l'impression que le Québec n'est plus le terrain d'enquête privilégié. Cinq rubriques structurent les numéros: Urbania, qui propose des réflexions de nature politique, Urania qui recueille des articles qui ne parlent pas du tout de politique, Ficciones, où l'on trouve des textes inédits de fiction, What I'm doing here, rubrique qui propose des réflexions sur les voyages et $\grave{A}$ la une, qui met en premier plan ce qui est vraiment important pour la rédaction. Les trois langues sont toujours utilisées par les auteurs des articles. Toutefois, certains numéros ne sont pas vraiment riches, car il n'y figure qu'un seul article, souvent de fiction. D'après ce que nous avons pu constater, il est possible de réagir et commenter les articles, et le commentaire va apparaître directement à la fin du texte avec le prénom et le nom de la personne qui a écrit. Il serait intéressant de voir également la nature des commentaires de la version en ligne, car en théorie, le magazine pourrait être lu partout et pourrait intéresser les diasporas du monde entier.

\section{BIBLIOGRAPHIE}

BERROUËT-Oriol Robert (1986), L'effet d'exil, Vice Versa, no 17, p. 20-21.

CACCIA Fulvio (dir.) (2010), La Transculture et Vice Versa: hier, aujourd'hui, demain, Montréal, Triptyque.

DAVAILLE Florence (2007), L'interculturalisme en revue : l'expérience de vice Versa, Voix et Images, vol. 32, n 2, p. 109-122.

DUMONTET Danielle (2014), La revue Vice Versa et le procès d'autonomisation des "écritures migrantes", Zeitschrift für Kanada-Studien, no 34, p. 87-104.

DUPUIS Gilles (2007), Redessiner la cartographie des écritures migrantes, Globe, vol. $10, \mathrm{n}^{\circ} 1$, p. 137-146. 
DuPUIS Gilles (2010), Vice et Versa, 10 ans déjà, Globe, vol. 13, n 2, p. 187-194.

HARZIMONT Michaël (2004), Le courrier des lecteurs. Entre co-construction du sens de l'événement contrôlée par le média et nécessaire prise en compte de l'usager $\mathrm{du}$ produit médiatique, Recherches en communication, $\mathrm{n}^{\circ} 21$, p. 27-41.

Mossetto Anna Paola (dir.) (2006), Le Projet transculturel de Vice Versa, Bologna, Edizioni Pendragon.

NAREAU Michel (2011), Une cartographie des revues culturelles au Québec, Globe, vol. 14, n ${ }^{\circ} 2$, p. 13-20.

NEPVEU Pierre (2006), "Vice Versa" ou la déstabilisation des lettres québécoises, in : Mossetto Anna Paola (dir), Le Projet transculturel de Vice Versa, Bologna, Edizioni Pendragon, p. 81-96.

TASSINARI Lamberto (1989), La Ville continue. Montréal et l'expérience transculturelle de Vice Versa, Community Development, $\mathrm{n}^{\circ}$ 21, p. 57-62.

TASSINARI Lamberto (2006), Sens de la transculture, in : MosSETTO Anna Paola (dir), Le Projet transculturel de Vice Versa, Bologna, Edizioni Pendragon, p. 17-30.

WILSON Sheena (2012), Multiculturalisme et transculturalisme : ce que peut nous apprendre la revue Vice Versa (1983-1996)», International Journal of Canadian Studies / Revue internationale d'études canadiennes, $\mathrm{n}^{\circ}$ 45-46, p. 261-275. 